Portland State University

PDXScholar

6-1-2010

\title{
Photoemission from Localized Surface Plasmons in Fractal Metal Nanostructures
}

Robert Campbell Word

Portland State University

Rolf Könenkamp

Portland State University, rkoe@pdx.edu

T. T. Dornan

Follow this and additional works at: https://pdxscholar.library.pdx.edu/phy_fac

Part of the Physics Commons

Let us know how access to this document benefits you.

Citation Details

Word, R. C., Dornan, T. T., \& Könenkamp, R. R. (2010). Photoemission from localized surface plasmons in fractal metal nanostructures. Applied Physics Letters, 96(25), 251110

This Article is brought to you for free and open access. It has been accepted for inclusion in Physics Faculty Publications and Presentations by an authorized administrator of PDXScholar. Please contact us if we can make this document more accessible: pdxscholar@pdx.edu. 


\title{
Photoemission from localized surface plasmons in fractal metal nanostructures
}

\author{
R. C. Word, T. Dornan, and R. Könenkamp ${ }^{\text {a) }}$ \\ Department of Physics, Portland State University, Portland, Oregon 97201, USA
}

(Received 9 April 2010; accepted 4 June 2010; published online 25 June 2010)

\begin{abstract}
We use photoemission microscopy to characterize localized surface plasmon distributions in nanostructured gold layers on indium-tin-oxide/glass substrates. The Au films have a fractal dimension of $\sim 1.3$ and smallest feature sizes of $\sim 100 \mathrm{~nm}$. We use femtosecond laser pulses at a wavelength of $\sim 800 \mathrm{~nm}$ for the plasmon excitation. Photoelectron emission occurs by a three-photon process in localized areas of indium-tin-oxide with $\sim 70 \mathrm{~nm}$ diameter. In these areas the photoemission rate is enhanced several thousand fold compared to nonstructured surface areas. The results show that plasmon enhanced photoemission can be induced in a nonabsorbing material in proximity to a plasmon-active metal nanostructure. (C) 2010 American Institute of Physics. [doi:10.1063/1.3457921]
\end{abstract}

Surface plasmons polaritons have attracted increased research interest due to their potential in concentrating and channeling light at the nanoscale. ${ }^{1,2}$ While plasmon excitations are typically propagating waves, they can be localized in metal films with nanostructured surface, in random geometries, and in islandic metal films. ${ }^{3,4}$ The localization occurs essentially by the confinement of local modes within the nanostructure. This type of localization can lead to a strong enhancement in the local plasmon intensity. "Hot spots" with optical intensities several orders of magnitude higher than the average background intensity have been observed, ${ }^{5,6}$ and are thought to be the source for surface-enhanced Raman scattering, enhanced luminescence, lasing, and other phenomena. Here we report on the possibility to utilize localized surface plasmons for enhanced nanoscale photoemission.

The most effective way to optically excite surface plasmons is by matching the wave vectors of the exciting light and the plasmon modes. In the localization regime the plasmon/light coupling is typically less efficient. ${ }^{3}$ We show in this paper that photoemission enhancement ${ }^{7,8}$ by several orders of magnitude can occur even under these conditions. We also show that plasmon-enhanced photoemission can occur outside the metallic material supporting the plasmon. We investigate a configuration where localized surface plasmons in gold lead to photoemission in indium-tin-oxide (ITO) which is transparent to the exciting light and lies in the proximity just outside the gold areas in which the light absorption occurs. Since we are able to resolve the size of the photoemission area, we can provide simple estimates for plasmon transport lengths and plasmon excitation efficiencies in these nanostructures.

$200 \mathrm{~nm}$ thick smooth $\mathrm{Au}$ films were prepared by vacuum evaporation of gold on to $80 \mathrm{~nm}$ thick conductive ITO-films on alkali-free glass slides of $0.2 \mathrm{~mm}$ thickness. After deposition, the Au films were Ga-milled in a FEI DB237 dualbeam focused ion beam system. Beam currents of $100 \mathrm{pA}$ and beam voltages of $30 \mathrm{kV}$, were used to mill areas of 10 $\times 10 \mu \mathrm{m}^{2}$ in size to prepare random nanostructures with various degrees of connectivity and feature size. Figure 1 shows results for this process obtained with exposure times of $180,170,160$, and $150 \mathrm{~s}$. The structure with the strongest photoemission is shown in Fig. 1(c) and has a gold coverage of $50 \%$, a Minkowski-Bouligand fractal dimension of 1.30, a gold border length of $600 \mu \mathrm{m}$, and a smallest feature size of $\sim 100 \mathrm{~nm}$. Typically 50 localized emission areas can be identified. Photoemission was also obtained in the other

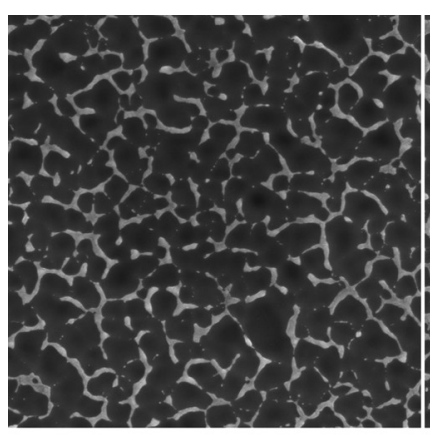

(a)

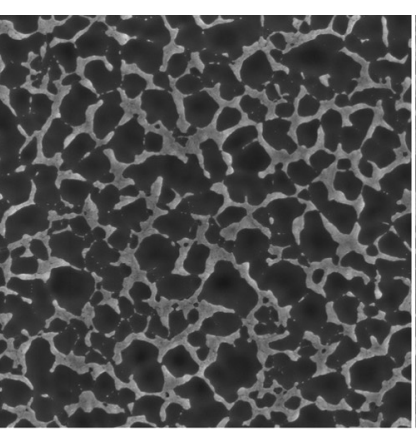

(b)

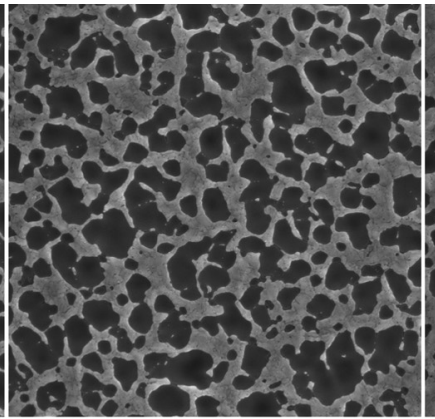

(c)

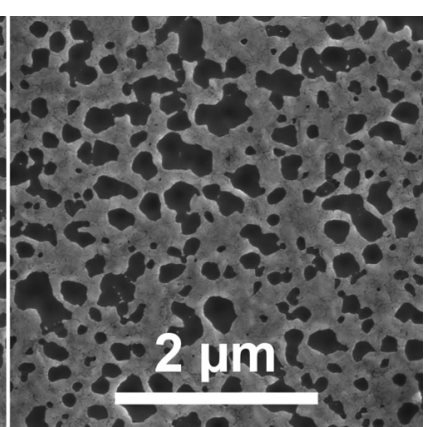

(d)

FIG. 1. Scanning electron micrographs of randomly structured Au films on ITO with Minkowski-Bouligand fractal dimension and Au coverage of (a) 1.36 and $21 \%$, (b) 1.35 and $33 \%$, (c) 1.30 and $50 \%$, and (d) 1.22 and $65 \%$, respectively. The sample in part (c) shows the strongest photoemission.

${ }^{a)}$ Electronic mail: rkoe@pdx.edu. 


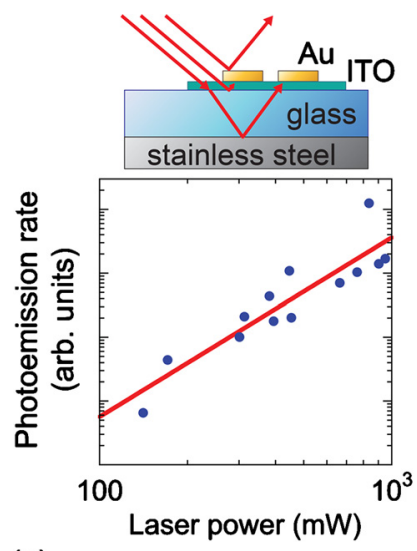

(a)

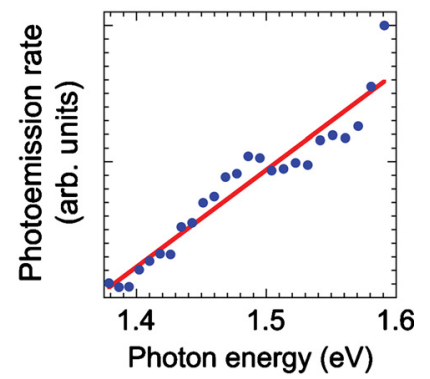

(b)

FIG. 2. (Color online) (a) Intensity dependence of the photoemission yield for $800 \mathrm{~nm}$ light. Inset: illumination scheme for nanostructured Au films on ITO. (b) Photon-energy dependence of the photoemission yield showing a threshold at $3 \times 1.37 \mathrm{eV}$.

structures shown in Fig. 1 but the emission rates and the number of emission areas are lower.

An aberration-corrected photoemission electron microscope (PEEM) (Ref. 9) was used to visualize the plasmon distribution. The microscope provides total-yield photoemission images employing a frequency-doubled $\mathrm{Ar}^{+}$-ion laser or an Hg lamp as ultraviolet light sources. With these sources the photoemission occurs in single-photon processes with linear intensity dependence. Plasmon excitation in the $\mathrm{Au}$ was achieved with a tunable Ti-sapphire laser providing 100 fs pulses at $80 \mathrm{MHz}$ at an angle of $60^{\circ}$ from the sample surface normal. Back and front side illumination of the $\mathrm{Au}$ layer occurs as indicated in Fig. 2(a). Simultaneous illumination by ultraviolet and infrared light allows a precise determination of the hot spot locations.

The tunable pulsed laser provides photon energies between 1.37 and $1.6 \mathrm{eV}$. Since the work functions for ITO and $\mathrm{Au}$ are significantly larger than these energies, multiplephoton processes are invoked to explain the observed photoemission. The indicator for multiphoton generation is a superlinear intensity dependence in the photoemission yield. Figure 2(b) shows the emission rate to be proportional to the third power of the laser intensity, indicating that a threephoton process is operative providing energies between 4.1 and $4.8 \mathrm{eV}$. As shown in Fig. 2(b) the photoemission has a threshold at $4.1 \mathrm{eV}$ which is consistent with the work function of ITO. ${ }^{10}$ Typical work function values for Au are 4.5 to $5.2 \mathrm{eV}$ depending on surface conditions. ${ }^{11}$ Thus photoemission from Au can only occur at the highest energies of the available range or in four-photon processes.

Figure 3 shows a close-up of the photoemission environment. In this particular example a bridge-type geometry is depicted where the planar ITO film connects two separate $\mathrm{Au}$ areas with a gap of $\sim 100 \mathrm{~nm}$. The composite micrograph clearly shows that the photoemission originates from the ITO film. Since the ITO itself is not absorbing at the primary photon energies, the photoemission must be induced by energy transfer from the absorbing Au film. The diameter of the emission area is typically $70 \pm 25 \mathrm{~nm}$ as shown in part (b). Comparing the count rates in the bright spots to count rates for unstructured planar areas of ITO films we find photoemission enhancement factors of $\sim 6000$ for the hot spots. Accounting for the cube-law in the three-photon process, the (a)
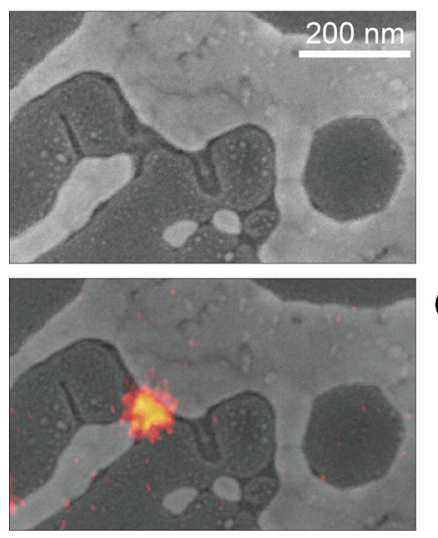

(b)

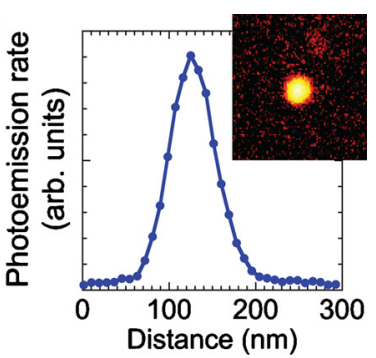

(c)

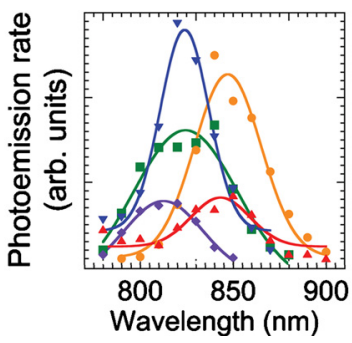

FIG. 3. (Color online) (a) Top: Scanning electron microsope (SEM) image of typical hot spot environment, bright areas are Au covered; bottom: composite micrograph of SEM and multiphoton PEEM images of localized photoemission at $\lambda=792 \mathrm{~nm}$ from ITO bridge between two $\mathrm{Au}$ islands. (b) Photoemission count rate across a localized photoemission area. Inset: PEEM image of localized emission. (c) Excitation spectra of various hot spots.

optical intensity enhancement comes out to be $\sim 18$.

The spectral properties of the localized plasmon resonances are shown in Fig. 3(c). From the excitation spectrum we find a typical bandwidth of $\sim 50 \mathrm{~nm}$, which gives quality factors of 16 to 18 . Thus both the q-factor and the intensity enhancement factor are of the same magnitude and consistent with the idea that the emissive areas are localized resonances of the plasmon distribution. The observed intensity enhancement of 18 in an area of $70 \mathrm{~nm}$ diameter could be achieved by energy collection over an area with a radius of approximately $150 \mathrm{~nm}$. Such a transport distance is consistent with the localized nature of the plasmon distribution as it corresponds to the smallest feature size in the Au layer structure. A transport distance of $150 \mathrm{~nm}$ also lies, as expected, at the very low end of transport ranges of non-localized surface plasmons in $\mathrm{Au}{ }^{12}$

Taking account of the instrument sensitivity we find that the average hot spot delivers only a few hundred electrons per second. The low emission rate can be attributed to the combination of inefficient light-to-plasmon coupling in the random geometry, low probabilities for the three-photon processes, and the short-range plasmon energy transfer. Clearly, all of these factors could be improved significantly. Thus, instead of the random geometry an efficient wave vector matching configuration could be utilized to improve the light coupling. With lower-work-function emitters and lower excitation wavelengths, two-photon processes, rather than threephoton processes could be utilized, and finally, by implementing improved plasmon transfer structures, larger enhancement factors could be achieved. With these improvements considerably more powerful electron sources should become feasible in planar geometry. At the present stage the main attractiveness lies in the fact that simple random structures experimentally permit nanoscale photoemission from low-energy photons. These structures may be useful in signal routing and processing, nanoelectrochemistry, and in nanometrology. For some of these applications it is of impor- 
tance that the electron emission can be induced in the nearfield proximity of metals, as we showed here.

To summarize, we have shown that locally enhanced photoemission can be obtained in random metal nanostructures. We estimate that the optical intensity in these hot spots is at least a factor $\sim 18$ higher than the intensity in unstructured films. This is consistent with plasmonic energy transfer distances of $\sim 150 \mathrm{~nm}$. The plasmon intensity enhancement translates into a photoemission enhancement of $6 \times 10^{3}$ due to the strong nonlinearity of a three-photon process. In the present study, photoemission is only observed outside the plasmon generating Au film in areas which are covered with ITO. The time-averaged photoemission rates are of the order of a hundred electrons per second. The experimental conditions are of interest for a number of useful applications of this technique in analysis and microscopy.

This work was supported by the Department of Energy Basic Science office under Grant No. DE-FG02-07ER46406.
T. Dornan acknowledges support from the Oregon Nano and Microtechnologies Institute, ONAMI.

${ }^{1}$ W. L. Barnes, A. Dereux, and T. W. Ebbesen, Nature (London) 424, 824 (2003).

${ }^{2}$ S. A. Maier and H. A. Atwater, J. Appl. Phys. 98, 011101 (2005).

${ }^{3}$ A. V. Zayats and I. I. Smolyaninov, J. Opt. A: Pure Appl. Opt. 5, S16 (2003).

${ }^{4}$ H. Raether, Springer Tracts Mod. Phys. 111, 1 (1988).

${ }^{5}$ M. Moskovits, Rev. Mod. Phys. 57, 783 (1985).

${ }^{6}$ M. I. Stockman, Phys. Rev. Lett. 84, 1011 (2000).

${ }^{7}$ H. Chen, J. Boneberg, and P. Leiderer, Phys. Rev. B 47, 9956 (1993).

${ }^{8}$ A. Gloskovskii, D. Valdaitsev, S. A. Nepijko, G. Schönhense, and B. Rethfeld, Surf. Sci. 601, 4706 (2007).

${ }^{9}$ R. Könenkamp, R. C. Word, G. F. Rempfer, T. Dixon, L. Almaraz, and T. Jones, Ultramicroscopy 110, 899 (2010).

${ }^{10}$ Y. Park, V. Choong, Y. Gao, B. R. Hsieh, and C. W. Tang, Appl. Phys. Lett. 68, 2699 (1996).

${ }^{11}$ J. C. Rivière, Appl. Phys. Lett. 8, 172 (1966).

${ }^{12}$ P. Andrew and W. L. Barnes, Science 306, 1002 (2004). 
Applied Physics Letters is copyrighted by the American Institute of Physics (AIP). Redistribution of journal material is subject to the AIP online journal license and/or AIP copyright. For more information, see http://ojps.aip.org/aplo/aplcr.jsp 